\title{
Development of New Microbiosensors
}

\author{
Isao KARUBE \\ Research Center for Advanced Science and Technology, \\ The University of Tokyo, \\ 4-6-1 Komaba, Meguro-ku, Tokyo 153, Japan
}

(Received November 5, 1990)

\begin{abstract}
ABTRACT: The miniaturization of biosensors is very promising in various field of applications. The authors have been developing many kinds of micro biosensors based on micro devices fabricated by semiconductor fabrication technology. Recently we have developed "ultra"-micro biosensors for in vivo monitoring. In this paper, we described the fabrication of micro biosensors which our group have developed.
\end{abstract}

KEY WORDS Micro Biosensor / ISFET / Micro Oxygen Electrode / Micro $\mathrm{H}_{2} \mathrm{O}_{2}$ Electrode / Integrated Micro Biosensors / Carbon Fiber Electrode

\section{Introduction}

The research on the biosensor development is being paid much attention. Conventional biosensors compose of electrochemical device, and immobilized biocatalysts. Oxygen, hydrogenperoxide and $\mathrm{pH}$ electrode have been mostly utilized as electrochemical devices of biosensors. However, with the increase of the demand of biosensor miniaturization, ISFETs and other micro electrodes which are produced by the semiconductor fabrication technology, are being utilized instead of common electrodes.

The miniaturization of biosensors has definetely advantages compared with the conventional biosensors. First, only tiny amount of sample is required for the measurement. Second, implantation of biosensor is possible, consequently enables in vivo measurement. Third, mass production of such microbiosensors is possible, consequently realizes disposable type biosensors. Fouth, various microbiosensors will be integrated to be constructed multi functionalized "integrated microbiosensors".

This review describes the current trends of microbiosensor development. 


\section{Potentiometric Microbiosensors}

\section{2-1 Microbiosensors using ISFET}

The ISFET(Ion Sensitive Field Effect Transistor) is a micro-pH device made by using silicon fabrication technology, and is attractive because of its small size and mass producibility. It was found as a micro-ion-sensitive device in proton, sodium and potassium ion and surface charge measurements. Therefore ISFET is useful for the potentiometric device of biosensors.

We developed a micro urea sensor using PVB immobilization method(1). As a result of this investigation, the following data were obtained. The differential gate output voltage reached a steady state approximately two minutes after injection. The reproducibility of the initial rate of the response curve was also measured. In particular, the response to $16.7 \mathrm{mM}$ urea was measured five times.

The urea sensor system gave a reproducible response with a coefficient of variance of $5.2 \%$. A linear relationship was obtained in the range of 1.3-16.7 $\mathrm{mM}$ urea. Slight responses were obtained when $6.3 \mathrm{mM}$ glucose, $10 \mathrm{mM}$ creatinine and $3.6 \mathrm{mM}$ albumin were applied to the system, even though the measurements were taken in a differential mode.

The immuno-FET sensor was also constructed by using ISFET and anti-human serum albumin (anti-HSA) immobilized PVB membrane(2). The measurements was based on the membrane potential change caused by immuno reaction of HSA and anti-HSA.

The differential gate output voltage change by binding the HSA to the anti-HSA was measured. The experiment was carried out at $\mathrm{pH}$ 7.0 and $37^{\circ} \mathrm{C}$. Since the isoelectric point of the HSA molecule is $\mathrm{pH}$ 4.8 , the HSA negative charge became bound to the anti-HSA, thereby altering the surface potential of the gate and producing a decrease in the gate output voltage.

The enzyme $\mathrm{H}^{+}$-ATPase catalyzes the hydrolysis of ATP to ADP and orthophosphate. A bioelectrochemical system for ATP determination based on ISFETs and immobilized H+-ATPase PVB membrane were developed based on the measurement of hydrogen ions generated by the enzyme reaction(3).

A novel biosensor system for acetylcholine and sodium ion influx consisting of the ISFET and the immobilized acetylcholine receptor PVB membrane was also constructed(4).

The sensor response was due to the positive charge of acetylcholine. A linear relationship was obtained between the initial rate of the differential gate output voltage change and the logarithmic value of the acetylcholine concentration in the range of 0.1-10 $\mu \mathrm{M}$. Immobilization of the acetylcholine receptor by using 
lipids was also performed. When the acetylcholine receptor was immobilized on a PVB-lipid membrane, the response was amplified with both the positive charge of acetylcholine and soldium ion influx through the acetylcholine receptor channel.

The development of simple and inexpensive assays for ethanol is important, with miniaturization of enzyme sensors particularly required in clinical fields. The cell membrane of acetic acidproducing bacteria has a complex enzyme system, therefore, can be used for application with an ISFET.

The authors have developed an alcohol-sensitive microbiosensor(alcohol-FET sensor) by use of an ISFET and the enzyme system existing in the cell membrane(5). The cell membrane was immobilized using calcium alginate gel and coated with nitrocellulose. Pyrrolequinoline quinone (PQQ) was added during the mixing of the cell membrane with sodium alginate. The PQQ concentration in the mixture was $0.5 \mathrm{mM}$.

A linear relationship was observed between the differential gate output and the ethanol concentration up to $20 \mathrm{mg} / 1$.

The substrate selectivity of the alcohol-FET sensor was examined. The sensor responded to ethanol, propan-1-ol, but did not respond to methanol, propan-2-ol and butan-2-ol.

\section{2-2 Microbiosensor using Amorphous Silicon ISFET (6)}

The conventional ISFET device can only be manufactured by using a silicon wafer as the substrate. Recently, devices made with amorphous silicon have been paid much attention. It was showed that the density in the gap for this material is sufficiently low to allow the material to be doped into n-type or p-type. Since then, extensive research on amorphous silicon and many devices has been carried out by many workers. Schottky barrier, metal-insulatorsemiconductor, pin (or nip) solar cells and charge-coupled devices have been fabricated with this material. Various substrates such as glass, plastics, etc. can be used for devices made with amorphous silicon and transistors can be fabricated with a number of different structures. The authours have developed an amorphous silicon ISFET (a-ISFET)

The device was mainly made by r.f. plasma discharge.

In order to use this device in water, the gate insulator was expected to have a role in preventing metal ions from penetrating the water solution. A silicon oxide layer was evaporated over an amorphous silicon nitride layer, acted as an insulator and a $\mathrm{pH}$ sensitive layer. The characteristics of the device as a $\mathrm{pH}$ sensor were then investigated. The linear $\mathrm{Vg} / \mathrm{pH}$ characteristics of an a-ISFET was obtained over the $\mathrm{pH}$ range 5-10. The $\mathrm{pH}$ sensitivity was about $46 \mathrm{mV} / \mathrm{pH}$ at $18^{\circ} \mathrm{C}$. A linear relationship was not obtained below 
$\mathrm{pH}$ 5.0. This phenomenon is mainly attributed to the density of surface-dissociated sites. On the other hand, it is thought that silicon oxide change to silicon dioxide partially. The $\mathrm{pH}$ characteristics of this device may also be due to changes in the $\mathrm{pH}$ sensitive layer. The response times of the a-ISFET to $\mathrm{pH}$ change were very rapid, less than $30 \mathrm{~s}$ being needed to reach a steady-state value. The long-term drift at $\mathrm{pH} 7.0$ was about $2.0 \mathrm{mV} / \mathrm{h}$. The origin of the drift may be attributed to the migration of some ion species in the gate insulator. This phenomenon may be due to the large threshold voltage of this device. In conclusion, our developed a-ISFET can be utilized as a $\mathrm{pH}$ sensor. With the use of a-ISFET, we developed microbiosensors.

Evaluation of fish freshness is important for the manufacture of high quality products. After fish die, decomposition of ATP (adenosine-5'-triphosphate), AMP (adenosine-5'-monophosphate) and related compounds are generated, consequently, hypoxanthine (Hx) accumulates with increase of storage time, and can be used as an indicator of fish meat freshness. The authors have previously reported on the fish freshness sensor using enzyme electrode. However, this sensor was complicated and required the combination of several enzyme electrodes. Therefore, simple and rapid methods for the determination of fish freshness are required by food industries.

Various methods have been described for hypoxanthine determinations, but they are complicated and time-consuming. An enzymatic method using xanthine oxidase (XO) has been applied for microbiosensor development. Generation of uric acid by the oxidation of hypoxanthine is detected based on an a-ISFET with immobilized xanthine oxidase(7). XO was immobilized onto the aISFET by the same method as the ISFET microbiosensors.

The sensor responses within $4 \mathrm{~min}$ after sample injection. This sensor exhibits a good linear relationship between the rate of change of the gate output voltage and logarithm of $\mathrm{Hx}$ concentration, over the range $0.02-0.1 \mathrm{mM}$.

As it was described above, inosine was also used as an indicator of fish freshness. Therefore, simple and rapid method for the determination of inosine levels in fish meats was developed(8). The calibration graph for inosine was linear over the range 0.02-0.1 $\mathrm{mM}$.

\section{Amperometric Microbiosensors}

3-1 Microbiosensors using micro-oxygen electrode

Many enzyme sensors and microbial sensors have been utilizing oxygen electrode as a transducer. Therefore the development of a 
micro-oxygen electrode will greatly contribute to the miniaturization of biosensors.

We currently developed an improved type micro-oxygen electrode based on conventional semiconductor fabrication technology(9).

The electrode has a U-formed groove, $300 \mu \mathrm{m}$ deep, and two gold electrodes over a $\mathrm{SiO}_{2}$ layer which electrically insulates them. Each gold electrode covers about half of the oxygen electrode. Agarose gels containing a $0.1 \mathrm{M}$ potassium chloride aqueous solution as an electrolyte were poured into the groove and then covered by the gas permeable membrane. The agarose layer was approximately 300 $\mu \mathrm{m}$ thick, and the gas permeable membrane was $2 \mu \mathrm{m}$ thick.

The micro-oxygen electrode was fabricated according to the conventional semiconductor fabrication process. The properties of this sensor as an oxygen electrode was measured. The $90 \%$ response time was approximately $3 \mathrm{~min}$, about three times longer than that of conventional clark-type oxygen electrode. This was probably due to the low oxygen diffusion through the agarose gel. The sensor response will be improved by shortening the distance between the cathode and the gas-permeable membrane. A linear relationship was obtained for an oxygen concentration between $1 \mathrm{ppm}$ and 7.9 ppm. These results indicated that the device could be employed as an oxygen sensor. We are also intending the application of microoxygen sensor to the fermentation process monitoring with colaboration of Prof. Armin Fiechter (ETH-Zurich).

Utilizing the micro-oxygen electrode, a micro-glucose sensor was constructed. The glucose sensor was constructed by immobilizing GOD on the sensing part of a micro-oxygen electrode by cross linking it with bovine serum albumin and glutaraldehyde. The glucose sensor responded as soon as the sample containing glucose was injected in the buffer solution, in which the sensing part of the micro-glucose sensor was dipped. A steady state current was observed after 5-10 min.

The sensor responded almost linearly for glucose concentrations between 0.2 and $2 \mathrm{mM}$. This sensitivity was comparable to conventional glucose sensors.

3-2 Microbiosensors using micro-gold electrodes

3-2-1 Micro-glucose sensor based on $\mathrm{H}_{2} \mathrm{O}_{2}$

measurement (10)

The enzyme sensor based on oxidase reaction can be constructed using an oxygen electrode or a $\mathrm{H}_{2} \mathrm{O}_{2}$ hydrogen peroxide electrode. We have also developed a micro- $\mathrm{H}_{2} \mathrm{O}_{2}$ hydrogen peroxide electrode. A micro-gold electrode was prepared on silicon nitride, using the 
vapor deposition method. Part of the gold electrode was coated with $\mathrm{Ta}_{2} \mathrm{O}_{5}$ for electrical insulation. This electrode works as a hydrogen peroxide sensor when the potential of both gold electrodes is set at $1.1 \mathrm{~V}$.

GOD was immobilized on an organic membrane prepared on the surface of a hydrogen peroxide sensor and constructed a microglucose sensor. A steady state current was obtained after $5 \mathrm{~min}$ of sample injection. A linear relationship was obtained between the current increase and the glucose concentration up to $10 \mathrm{mg} / \mathrm{dL}$.

3-2-2 Micro-glucose sensor utilizing artificial electron mediator. (13)

Glucose sensors based on the measurement of dissolved oxygen or hydrogen peroxide have problems considering their application to the in vivo monitoring in whole blood and plasma. The dynamic range of glucose determination decreases by the lack of dissolved oxygen. Normally, glucose concentration in human blood is appoximately 4-6 $\mathrm{mM}$, but in the diabetes' patients it is more than $30 \mathrm{mM}$. However, dissolved oxygen in blood is normally $2.2 \mathrm{mM}$. and it is insufficient to oxidize total glucose. Artificial electron mediators have been used to overcome these disadvantages. Increasing the amount of mediator in the solution or enzyme immobilizing membrane, dynamic range will be expanded.

Polypyrolle (PPy) has recently been the subject of much attention because of its excellent properties.

We developed a novel method to immobilize both GOD and electron mediator on to the micro-electrode. This method was applied for the construction of a micro-glucose sensor. We used dimethylferrocene (DMFe) as the electron mediator. As a transducer, a micro-gold electrode was utilized. A PPy modified electrode was prepared by electropolymerizing in an aqueous solution of $0.1 \mathrm{M} \mathrm{KCl}$ for $2 \mathrm{~min}$ at room temperature. GOD was immobilized by adsorbing on PPy films prepared on to the surface of a gold electrode as follows. The PPy modified electrode was dipped into a GOD aqueous solution, overnight at $4^{\circ} \mathrm{C}$. The electrode was then rinsed in distilled water and dried in a desiccator. The GOD adsorbed electrode was dipped into dichlorolethane solution containing $1 \% \mathrm{DMFe}$ and $2 \%$ polyvinylbutyral (PVB), and then dried in the air. Therefore, DMFe was succesively entrapped into the PVB matrix.

This micro-glucose sensor utilizing DMFe was operated potentiostatically at $0.1 \mathrm{~V}$ in a nitrogen gas buffer. The results indicated that this sensor's dynamic range was very wide $(2.5-30$ $\mathrm{mM}$ ), and sufficient for the determination of glucose concentration in vivo. 
4. Integrated Microbiosensors

4-1 Integrated glucose sensor

Simultaneous determination of glucose and oxygen for the in vivo monitoring is required not only because oxygen concentration monitoring in blood is necessary for understanding the respiratory state of a patient, but also because the glucose sensor response is affected by oxygen concentration. In order to realize such simultaneous monitoring, a glucose sensor and an oxygen electrode were integrated on a glass substrate (12).

The sensor consisted of a counter electrode and four working electrode (two of these were used in this case). All of these electrodes were formed by depositing gold on top of chromium on a Corning 7059 glass substrate in high vacuum conditions. GOD was immobilized on the electropolymerized polypyrrole film of a working electrode using BSA and GA. DMFe was incorporated by dipping the GOD-immobilized part into a DMFe-dissolved chloroform or acetone solution. Another working electrode was used as an oxygen electrode. A $\mathrm{Ag} / \mathrm{AgCl}$ electrode was used as a reference electrode. The same counter and reference were used for both the glucose sensor and the oxygen electrode were maintained at $+0.1 \mathrm{~V}$ and $-0.3 \mathrm{~V}$ versus $\mathrm{Ag} / \mathrm{AgCl}$, respectively.

The responses of the glucose sensor and the oxygen electrode were evaluated simultaneously in a 0.1-M phosphate buffer ( $\mathrm{pH} 7.0,30$ $\left.{ }^{\circ} \mathrm{C}\right)$. The response time for the glucose sensor was approximately one minute. The glucose sensor and the oxygen electrode responded independently and the oxygen electrode could be used to calibrate the glucose sensor.

4-2 Integrated microbiosensor for the simultaneous measurement of glucose and galactose(13)

Glucose oxidation via quinoprotein glucose dehydrogenase (GDH) occurs by the electron transfer between glucose and pyrrolequinolinequinone (PQQ) in the GDH. This electron transfer is not affected by dissolved oxygen. Therefore, a glucose sensor which is not affected by dissolved oxygen was constructed by utilizing this GDH.

We recently developed an integrated microbiosensor for the simultaneous measurement of glucose and galactose. We immobilized quinoprotein GDH and galactose oxidase (GAOD) onto a microelectrode. The concentration of glucose was determined by using electron mediator, and those of galactose was determined by measuring dissolved oxygen. The utilized quinoprotein GDH was purified from Pseudomonas fluorescens, which was kindly provided 
by Prof. Ameyama (Yamaguchi Univ., Japan ). The integrated microbiosensor was constructed by casting a GDH and GAOD BSA solution onto a microelectrode, and crosslinkihg with GA. In this experiment, ferrocene monocarbonate(FCA) was dissolved in the reaction buffer, and utilized as an artificial electron mediator. The terminal voltage of the sensor measuring FCA was set at $+0.35 \mathrm{mV}$ and those of the sensor measuring dissolved oxygen was set at -0.3 $\mathrm{mV}$ vs. $\mathrm{Ag} / \mathrm{AgCl}$ respectively.

The determination of galactose concentration by this sensor was first attempted. Since GAOD was also catalyzed by the electron transfer to FCA, both electrode showed the response to galactose. With the increase of galactose concentration, the response toward glucose decreased. However, by comparing with the sensor response to galactose measuring dissolved oxygen, the appropriate calibration curve can be chosen. Therefore, the simultaneous measurement of glucose and galactose can be performed by utilizing this integrated microbiosensor.

\section{Ultra-micro biosensors}

Recent studies have demonstrated that an in vivo electrochemical electrode is a powerful tool for biomedical and clinical monitoring. It is desirable that such a sensor is much smaller than conventional micro biosensors.

Carbon fiber was considered to be useful as an ultra micro electrode. $\mathrm{H}_{2} \mathrm{O}_{2}$ detection is important in fabrication of biosensors because some immobilized-enzymes produce $\mathrm{H}_{2} \mathrm{O}_{2}$ as products. It is, however, difficult to measure $\mathrm{H}_{2} \mathrm{O}_{2}$ accurately by electrochemical techniques since it has an irreversible and irreproducible reaction at various electrodes. Therefore exploration of the electrode material or improvement of the electrode surface is necessary to decrease the overpotential of $\mathrm{H}_{2} \mathrm{O}_{2}$ oxidation or reduction. A carbon electrode appears promising to improve and define better its surface structure for the anodic oxidation of $\mathrm{H}_{2} \mathrm{O}_{2}$. The length of the electrode we used was $500 \mu \mathrm{m}$ and the diameter of the electrode was $7 \mu \mathrm{m}$. Epoxy resin was used for the shield and silver paste for the electrical contact.

An activation signal which is an anodic-cathodic triangular wave was introduced immediately before every measuring pulse. The parameters for measuring pulses were decided as follows. The length of the first pulse $(750 \mathrm{mV})$ was $1000 \mathrm{msec}$. At the end of the first pulse, the current was measured every $625 \mu \mathrm{s}$ for $160 \mathrm{~ms}$, turns out 256 measurement points and then the average was obtained from 256 current values. The length of the second pulse 
was $1000 \mathrm{~ms}$. The polarization potential changes from 750 to $1100 \mathrm{mV}$. The current is measured every 625 us for $160 \mathrm{~ms}$, which is one cycle of the electric line $(50$ cycles/s). The 32 measured data were added, which was some sort of average. These procedures enabled sensitivity and first and second pulse were measured by microcomputer-controlled potentiostat system after injection of $\mathrm{H}_{2} \mathrm{O}_{2}$.

A good relationship was observed between the current change and $\mathrm{H}_{2} \mathrm{O}_{2}$ concentration. The detection limit is less than $0.1 \mu \mathrm{M}$. By the introduction of the electrochemical treatment before every measurement pulse, the sensitivity and reproducibility were remarkably improved. The separation of $\mathrm{H}_{2} \mathrm{O}_{2}$ from ascorbic acid was possible even in solutions containing albumin proteins.

Immobilized enzyme was prepared on the surface of a carbon fiber electrode. Glucose oxidase was used as a molecular recognition part of the biosensor. A linear calibration was obtained for the glucose concentration.

\section{References}

1. M.Gotoh, E.Tamiya and I.Karube, Sensors and Materials, 1,25 33(1988)

2. M.Gotoh, E.Tamiya and I.Karube, Nippon Kagaku Kaishi, 11, 2214 - 2221(1987)

3. M.Gotoh, E.Tamiya and I.Karube, Anal.Chim.Acta, 187, 287 291(1986)

4. M.Gotoh, E.Tamiya, M.Momoi, Y.Kagawa and I.Karube, Anal.Lett., 20, 857 - 870(1987)

5. E.Tamiya, I.Karube, Y.Kitagawa, M.Ameyama and K.Nakashima, Anal.Chim.Acta, 207, 77 - 84(1988)

6. M.Gotoh, S.Oda, I.Shimizu, A.Seki, E.Tamiya and I.Karube, Sensors and Actuators, 16, 55 - 65(1989)

7. E.Tamiya, A.Seki, I.Karube, M.Gotoh and I.Shimizu, Anal.Chim.Acta, 215, $301-305(1988)$

8. M.Gotoh, E.Tamiya, A.Seki, I.Shimizu and I.Karube, Anal.Lett., 21, 1785 - 1800(1988)

9. H.Suzuki, E.Tamiya and I.Karube, Anal.Chem., 60, 1078 1080(1988)

10. I.Karube, E.Tamiya, T.Murakami, M.Gotoh and Y.Kagawa, Enz.Eng.8, Ann.N.Y.Acad.Sci., 501, 256 - 264(1987)

11. E.Tamiya, S.Hattori, M.Suzuki, K.Yokoyama, and I.Karube, Sensors and Actuators, 18, 297-307 (1988)

12. K.Yokoyama, E.Tamiya and I.Karube, Anal.Lett., 22, 2949-2959

13. K.Yokoyama, K.Sode, E.Tamiya and I.Karube, Anal.Chim.Acta, 218, 137 - 142(1989) 